\title{
THE KUNZ-SOUILLARD APPROACH TO LOCALIZATION FOR JACOBI OPERATORS
}

\begin{abstract}
VALMIR BUCAJ
Abstract. In this paper we study spectral properties of Jacobi operators. In particular, we prove two main results: (1) that perturbing the diagonal coefficients of Jacobi operator, in an appropriate sense, results in exponential localization, and purely pure point spectrum with exponentially decaying eigenfunctions; and (2) we present examples of decaying potentials $b_{n}$ such that the corresponding Jacobi operator has purely pure point spectrum.
\end{abstract}

Mathematics subject classification (2010): 47B37.

Keywords and phrases: Jacobi operators, decaying potentials, spectrum.

\section{REFERENCES}

[1] M. Aizenman, S. Molchanov, Localization at large disorder and at extreme energies: an elementary derivation, Comm. Math. Phys. 157 (1993), 245-278.

[2] V. Bucaj, D. Damanik, J. Fillman, V. Gerbuz, T. VandenBoom, F. Wang, Z. Zhang, Localization for the one dimensional Anderson model via positivity and large deviation for the Lyapunov exponent, arXiv:1706.06135 (2017).

[3] V. BUCAJ, On the Kunz-Souillard approach to localization for the discrete one dimensional generalized Anderson model, arXiv: 1608.01379, 24 Oct. 2016.

[4] H. L. Cycon, R. G. Froese, W. Kirsch, B. Simon, Schrödinger operators with applications to quantum mechanics and global geometry, Texts and Monographs in Physics, Springer Study Edition (1987).

[5] D. DamaniK, G. Stolz, A continuum version of the Kunz-Souillard approach to localization in one dimension, J. Reine Angew. Math. 660 (2011), 99-130.

[6] D. DamaniK and J. Fillman, Spectral Theory of Discrete One-Dimensional Ergodic Schrödinger Operators, preprint.

[7] D. Damanik, A. Gorodets KI, An extension of the Kunz-Souillard approach to localization in one dimension and applications to almost-periodic Schrödinger operatos, Advances in Mathematics, vol. 297 (2016), pp. 149-173.

[8] D. DamaniK, A Short Course on One-Dimensional Random Schrödinger Operators, arXiv:1107.1094v1, 6 Jul. 2011, preprint.

[9] F. Delyon, H. Kunz, B. Souillard, One-Dimensional Wave Equations in Disordered Media, J. Phys. A: Math. Gen. 16 (1983) 25-42.

[10] M. S. P. EAstham, The spectral theory of periodic differential equations, Texts in Mathematics, Edinburgh-London: Scottish Academic Press, (1973).

[11] J. FRÖHLICH, T. SPENCER, Absence of diffusion in teh Anderson tight binding model for large disorder or low energy, Comm. Math. Phys. 88 (1983), 151-184.

[12] W. KIRsch, An Invitation to Random Schrödinger Operators, Panor. Synthéses 25, Random Schrödinger operators, 1-119, Soc. Math. France, Paris, 2008.

[13] H. KunZ, B. SOUILlaRd, Sur le spectre des opérateurs aux différences finies aléatoires, Commun. Math. Phys. 78, (1980), 201-246.

[14] S. JitOMIRSKAYA, H. SCHUlZ-BLADES, Upper bounds on wavepacket spreading for random Jacobi matrices, Commun. Math. Phys. 273 (2007), 601-618. 
[15] S. Jitomirskaya, H. Schulz-Blades, G. Stolz, Delocalization in random polymer models, Commun. Math. Phys. 233 (2003), 27-48.

[16] M. Reed, B. Simon, Methods of modern mathematical physics. IV: Analysis of operators, New York-San Francisco-London: Academic Press (1978).

[17] R. DEL Rio, S. JitomirS KAYA, Y. LASt, B. Simon, Operators with singular continuous spectrum. IV. Hausdorff dimensions, rank one pertubations, and localization, J. Anal. Math. 69 (1996), 153-200.

[18] B. Simon, Some Jacobi Matrices with Decaying Potential and Dense Point Spectrum, Commun. Math. Phys. 87, (1982) 253-258.

[19] B. Simon, Szegö's Theorem and Its Descendants: Spectral Theory for $L^{2}$ Pertubations of Orthogonal Polynomials, Princeton University Press, (2010).

[20] G. StolZ, An Introduction to the Mathematics of Anderson Localization, Contemp. Math. 552 (2011), 71-108.

[21] G. Techl, Mathematical Methods in Quantum Mechanics: With Applications to Schrödinger Operators, Grad. Stud. Math, Vol. 99, 2009. 